\title{
THE CARTESIAN CLOSED BICATEGORY OF GENERALISED SPECIES OF STRUCTURES
}

\author{
M. FIORE, N. GAMBINO, M. HYLAND, AND G. WINSKEL
}

\begin{abstract}
The concept of generalised species of structures between small categories and, correspondingly, that of generalised analytic functor between presheaf categories are introduced. An operation of substitution for generalised species, which is the counterpart to the composition of generalised analytic functors, is also put forward. These definitions encompass most notions of combinatorial species considered in the literature - including of course Joyal's original notion - together with their associated substitution operation. Our first main result exhibits the substitution calculus of generalised species as arising from a Kleisli bicategory for a pseudo-comonad on profunctors. Our second main result establishes that the bicategory of generalised species of structures is cartesian closed.
\end{abstract}

\section{INTRODUCTION}

The concept of species of structures, introduced by Joyal [23], is a fundamental notion in modern enumerative combinatorics. A species of structures describes precisely what is informally understood by a type of labelled combinatorial structure, and can be regarded as a structural counterpart of a counting formal exponential power series. To provide a satisfactory conceptual basis for the theory of species of structures, Joyal [24] also introduced the theory of analytic functors. Analytic functors can be regarded as structural counterparts of exponential generating functions, and provide an equivalent view of species of structures as Taylor series. The theory of species of structures $[23,24,7]$ provides a rich calculus that is a structural counterpart of the calculus of formal exponential power series. Among other things, this calculus explains formal combinatorial counting arguments by means of bijective proofs. For such and other applications of the theory of species in combinatorics the reader is referred to [7].

One of the fundamental operations on combinatorial species is that of substitution. Indeed, for a whole variety of notions of combinatorial species, operations of substitution have been defined. Each of these corresponds to the composition of associated formal power series (see, e.g., [23, 24, 40, 6, 38, $37,7]$ ). In particular, Joyal [24] showed that the operation of substitution for

Key words and phrases. Species of structures, analytic functor, bicategory, cartesian closed structure. 
species of structures corresponds to the functorial composition of analytic functors.

Our first aim here is to give a general and uniform treatment of combinatorial species and their substitution operation. After recalling the basic theory of species of structures and analytic functors, we introduce the more general concepts of species of structures between small categories and of analytic functors between presheaf categories; the former being Taylor series of the latter. Generalised species are then equipped with an operation of substitution akin to the various existing notions for combinatorial species. Our definition is shown to correspond to the series of Taylor coefficients of the functorial composition of generalised analytic functors. This leads to the definition of a bicategory of generalised species of structures. This material constitutes the first part of the paper, and comprises Sections 2 and 3.

The substitution operation on species of structures underlies a well-known and important monoidal structure introduced by Kelly [25] (see also [27, 28]). Indeed, the monoids for this substitution monoidal structure are the symmetric set-operads of May [35]. More recently, Baez and Dolan considered further generalisations of these structures leading to the concepts of sorted symmetric set-operad [1] and of stuff types [2] (see also [39]). The former, though not the latter, can be directly recast in our setting: the substitution monoidal structures arise from the endo-homs of the bicategory of generalised species, and the operads are the monads in there.

Our second aim is twofold: to give an abstract theory of the substitution calculus of generalised species and to further enrich the calculus of combinatorial species by adding a new dimension to it. To this end, we introduce a pseudo-comonad whose Kleisli bicategory has generalised species as morphisms, with composition amounting to substitution. The Kleisli bicategory of generalised species of structures is then shown to be cartesian closed. Hence, it does not only supports operations for pairing and projecting, which are implicit in the combinatorial literature, but also operations of abstraction and evaluation, yet to be exploited in combinatorics. This development is presented in the second part of the paper, which comprises Sections 4 and 5. Applications are discussed in Section 6.

The construction leading to the Kleisli bicategory of generalised species is analogous to the construction of the relational model of linear logic [20]. This model can be seen to arise by observing that the monad on the category of sets whose algebras are commutative monoids extends to a monad on the category of relations via a distributive law. Using the duality available on the category of relations, this monad can be turned into a comonad that has the properties of a linear exponential comonad and thus determines a cartesian closed category via the Kleisli construction (see, e.g., [36, 41]). Our work demonstrates that it is possible to carry over a similar programme in the context of 2-dimensional category theory; with the 2-category of small 
categories replacing the category of sets, the bicategory of profunctors replacing the category of relations, the 2-monad for symmetric [strict] monoidal categories replacing the monad for commutative monoids, and the duality on profunctors replacing the duality on relations. This viewpoint has also been considered in [10], where cartesian closed structures of the type described here were indicated informally. Within the context of linear logic, our model generalises and extends a model that was a precursor to models of linear logic: the model of normal functors (viz. generating functors of formal set-valued multivariate power series) introduced by Girard [21] to construct a model of the lambda calculus. Within the context of 2-dimensional algebra, our construction of the Kleisli bicategory is similar to an abstract construction of Day and Street in [14, Section 2]; a main difference being that they consider a pseudo-comonad associated to the free (non-commutative) monoid construction, which in our setting would amount to considering the free (non-symmetric) strict monoidal completion.

For the convenience of the reader, relevant notions of 2-dimensional category theory are recalled in Appendix A. Throughout the paper, we use well-known properties of presheaf categories and make extensive use of the calculus of coends, for which the reader is referred to [33, Chapter X]. For background on monoidal categories, see e.g. $[16,32]$.

\section{Species of Structures And ANALYTiC FunCtors}

2.1. Species of structures. Recall from [23] that a species of structures is a functor $\mathbf{B} \rightarrow$ Set, for $\mathbf{B}$ the groupoid of finite sets and bijections, and Set the category of sets and functions. Species of structures are essentially determined by their action on finite cardinals. To express this precisely, let $\mathbf{P}$ be the full subgroupoid of $\mathbf{B}$ whose objects are the finite cardinals $[n]=_{\text {def }}\{i \in \mathbb{N} \mid 1 \leq i \leq n\}$, with $n \in \mathbb{N}$. Thus, the hom-sets of $\mathbf{P}$ are as follows:

$$
\mathbf{P}[u, v]={ }_{\operatorname{def}}\left\{\begin{array}{cl}
\mathbf{P}_{n} & , \text { if } u=v=[n] \\
\emptyset & , \text { otherwise }
\end{array}\right.
$$

where $\mathbf{P}_{n}$ denotes the symmetric group on $[n]$. Then, composition with the embedding $\mathbf{P} \hookrightarrow \mathbf{B}$ yields an equivalence of functor categories

$$
[\mathbf{B}, \text { Set }] \stackrel{\simeq}{\longrightarrow}[\mathbf{P}, \text { Set }] \text {. }
$$

In view of the above, and without loss of generality, we will henceforth consider species of structures either as functors $\mathbf{B} \rightarrow$ Set or $\mathbf{P} \rightarrow$ Set depending on the point of view that is most convenient.

2.2. Analytic functors and substitution. Every species of structures $F$ determines an endofunctor $\widetilde{F}:$ Set $\rightarrow$ Set, called the analytic functor associated to $F$. For $F: \mathbf{P} \rightarrow$ Set, the endofunctor $\widetilde{F}$ is a left Kan extension 
of $F$ along the inclusion $\mathbf{P} \rightarrow$ Set

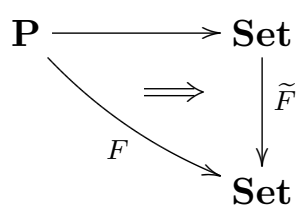

and we define it as follows:

$$
\widetilde{F}(X)=\operatorname{def} \int^{u \in \mathbf{P}} F(u) \times X^{u} .
$$

Note however that this can be simplified to yield a description as an exponential power series. Indeed,

$$
\widetilde{F}(X) \cong \sum_{n \in \mathbb{N}} F[n] \times X^{n} / \mathbf{P}_{n}
$$

where $F[n] \times X^{n} / \mathbf{P}_{n}$ is the quotient of $F[n] \times X^{n}$ induced by the left $\mathbf{P}_{n}$-action on $F[n]$ given by the action of $F$ and the right $\mathbf{P}_{n}$-action on $X^{n}$ given by permuting $n$-tuples. In general, an endofunctor on Set is said to be analytic [24] whenever it is naturally isomorphic to the analytic functor associated to a species. Moreover, whenever $T \cong \widetilde{F}$, one says that $F$ is the species of Taylor coefficients of the analytic functor $T$; the concept being well-defined up to natural isomorphism [24, Theorem 1].

It is a remarkable fact that analytic functors are closed under composition. This can be seen to follow from the fact that there is an operation of substitution [24] that to every pair of species $F, G$ associates a species $G \circ F$ such that

$$
\widetilde{G \circ F} \cong \widetilde{G} \widetilde{F} .
$$

As explained in [7, Section 1.4], the substitution operation for species of structures is a structural counterpart to the composition of formal exponential power series.

To define substitution we need recall that the groupoid $\mathbf{P}$ has a canonical symmetric strict monoidal structure whose unit is the empty cardinal and whose tensor product, here denoted $\oplus$, is given by the addition of cardinals. The symmetry is given by the natural isomorphism whose components are the bijections

$$
[m] \oplus[n]=[m+n] \underset{\sigma_{m, n}}{\cong}[n+m]=[n] \oplus[m]
$$

defined by

$$
\sigma_{m, n}(k)=1+((k+n-1) \bmod (m+n)) .
$$

Then, in the vein of [25], the substitution operation can be defined as follows:

$$
(G \circ F)(u)=\operatorname{def} \int^{v \in \mathbf{P}} G(v) \times F^{* v}(u)
$$


where

$$
F^{*[n]}(u)=_{\operatorname{def}} \int^{u_{1}, \ldots, u_{n} \in \mathbf{P}} \prod_{i \in[n]} F\left(u_{i}\right) \times \mathbf{P}\left[\oplus_{i \in[n]} u_{i}, u\right] .
$$

For a proof of (2) see [24, Section 2.1 (iv)], or Theorem 3.2.1 below.

Finally, we recall that the substitution operation underlies an important monoidal structure on the category of species of structures; see [25]. The unit $I$ for the substitution tensor product is characterised by the fact that $\widetilde{I} \cong \operatorname{Id}_{\text {Set }}$, and is defined as

$$
I[n]=\operatorname{def}\left\{\begin{array}{cl}
{[1]} & , \text { if } n=1 \\
\emptyset & , \text { otherwise }
\end{array}\right.
$$

\section{Generalised species of Structures And Analytic functors}

3.1. Generalised species of structures. Our generalisation of the notion of species of structures combines two ideas. The first idea is to generalise from the category $\mathbf{B}$ to a category $\mathbf{B} A$, parameterised by a small category $A$, such that $\mathbf{B} \mathbf{1} \cong \mathbf{B}$. This construction already occurs in [23, Section 7.3], and is given as follows: the objects of $\mathbf{B} A$ are families $\left\langle a_{i}\right\rangle_{i \in I}$ with $I \in \mathbf{B}$ and $a_{i} \in A$; a morphism $\left\langle a_{i}\right\rangle_{i \in I} \longrightarrow\left\langle a_{j}^{\prime}\right\rangle_{j \in J}$ in $\mathbf{B} A$ consists of a pair $\left(\sigma,\left\langle f_{i}\right\rangle_{i \in I}\right)$ with $\sigma: I \longrightarrow \cong$ in $\mathbf{B}$ and $f_{i}: a_{i} \rightarrow a_{\sigma(i)}^{\prime}$ in $A$. Composition and identities are the obvious ones. The second idea is to generalise from Set to presheaf categories on small categories, i.e. functor categories of the form $\widehat{B}={ }_{\text {def }}\left[B^{\circ}\right.$, Set $]$ with $B$ small.

The following definition generalises the concept of species of structures, which thus arises as that of $(\mathbf{1}, \mathbf{1})$-species of structures. Further examples of combinatorial species subsumed by our generalisation are mentioned in Section 6.1.

3.1.1. Definition. An $(A, B)$-species of structures between small categories $A$ and $B$ is a functor $\mathbf{B} A \rightarrow \widehat{B}$.

Note that, analogously to what happens with standard species of structures, generalised species can be equivalently defined by restricting $\mathbf{B} A$ to its full subcategory $\mathbf{P} A$ consisting of sequences, i.e. families indexed by finite cardinals. Indeed, restriction along the embedding $\mathbf{P} A \hookrightarrow \mathbf{B} A$ determines, for all small categories $A$ and $B$, an equivalence of functor categories

$$
[\mathbf{B} A, \widehat{B}] \stackrel{\simeq}{\longrightarrow}[\mathbf{P} A, \widehat{B}]
$$

a quasi-inverse to which is given by the mapping that left Kan extends along the embedding $\mathbf{P} A \hookrightarrow \mathbf{B} A$.

In view of the above, we will henceforth consider $(A, B)$-species of structures either as functors $\mathbf{B} A \rightarrow \widehat{B}$ or $\mathbf{P} A \rightarrow \widehat{B}$ depending on the point of view that is most convenient. 
3.2. Generalised analytic functors and substitution. Generalising the situation for species of structures, we consider analytic functors $\widetilde{F}: \widehat{A} \rightarrow \widehat{B}$ associated to $(A, B)$-species of structures $F$. Consequently, a functor between presheaf categories is said to be analytic whenever it is naturally isomorphic to the analytic functor associated to a generalised species. As in the case of standard analytic endofunctors on Set, also in this general context one is justified to refer to $F$ as the generalised species of Taylor coefficients of any analytic functor naturally isomorphic to $\widetilde{F}$.

For $F: \mathbf{P} A \longrightarrow \widehat{B}$, the functor $\widetilde{F}: \widehat{A} \rightarrow \widehat{B}$ is given as follows:

$$
\widetilde{F}(X)_{b}={ }_{\operatorname{def}} \int^{u \in \mathbf{P} A} F(u)_{b} \times X^{u}
$$

where

$$
X^{\left\langle a_{i}\right\rangle_{i \in[n]}}={ }_{\operatorname{def}} \prod_{i \in[n]} X_{a_{i}} .
$$

Note that $\widetilde{F}$ is a left Kan extension of $F$

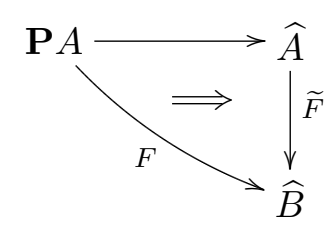

along the sum functor $\mathrm{S}_{A}: \mathbf{P} A \rightarrow \widehat{A}$ given by

$$
\mathrm{S}_{A}\left(\left\langle a_{i}\right\rangle_{i \in[n]}\right)={ }_{\operatorname{def}} \sum_{i \in[n]} \mathrm{y}_{A}\left(a_{i}\right),
$$

where $\mathrm{y}_{A}$ denotes the Yoneda embedding $A \hookrightarrow \widehat{A}$. Indeed, this readily follows by observing that

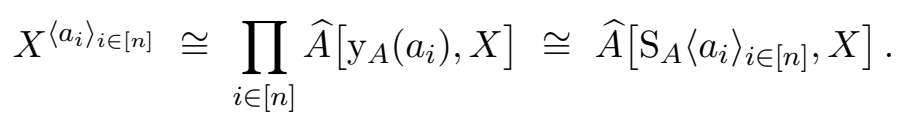

Further note that, for $n \in \mathbb{N}$ and $u_{j} \in \mathbf{P} A(j \in[n])$, there are canonical coherent natural isomorphisms as follows:

$$
\sum_{j \in[n]} \mathrm{S}_{A}\left(u_{j}\right) \cong \mathrm{S}_{A}\left(\oplus_{j \in[n]} u_{j}\right)
$$

We now introduce an operation of substitution for generalised species of structures that generalises (4). To give the definition we need to consider a canonical symmetric strict monoidal structure on $\mathbf{P} A$. The unit is the empty sequence, denoted \langle\rangle . The tensor product, denoted $\oplus$, is given by sequence concatenation. Explicitly,

$$
\left\langle a_{i}\right\rangle_{i \in[m]} \oplus\left\langle a_{j}^{\prime}\right\rangle_{j \in[n]}=_{\text {def }}\left\langle\left[a, a^{\prime}\right]_{k}\right\rangle_{k \in[m+n]}
$$

where

$$
\left[a, a^{\prime}\right]_{k}= \begin{cases}a_{k} & , \text { for } 1 \leq k \leq m \\ a_{k-m}^{\prime} & , \text { for } m+1 \leq k \leq m+n\end{cases}
$$


The symmetry has components

$$
\left\langle\left[a, a^{\prime}\right]_{k}\right\rangle_{k \in[m+n]} \frac{\left(\sigma_{m, n},\left\langle\operatorname{id}_{\left[a, a^{\prime}\right]_{k}}\right\rangle_{k \in[m+n]}\right)}{\cong}\left\langle\left[a^{\prime}, a\right]_{k}\right\rangle_{k \in[n+m]}
$$

where $\sigma_{m, n}$ is the component of the symmetry of $\mathbf{P}$ defined in (3).

For an $(A, B)$-species $F$ and a $(B, C)$-species $G$, the substitution $(A, C)$-species $G \circ F$ is defined as follows:

$$
(G \circ F)(u)_{c}=_{\operatorname{def}} \int^{v \in \mathbf{P} B} G(v)_{c} \times F^{* v}(u)
$$

where

$$
F^{*\left\langle b_{i}\right\rangle_{i \in[n]}}(u)={ }_{\operatorname{def}} \int^{u_{1}, \ldots, u_{n} \in \mathbf{P} A} \prod_{i \in[n]} F\left(u_{i}\right)_{b_{i}} \times \mathbf{P} A\left[\oplus_{i \in[n]} u_{i}, u\right] .
$$

The next theorem implies that generalised analytic functors are closed under composition.

3.2.1. Theorem. The analytic functor associated to a substitution generalised species is naturally isomorphic to the composite of the analytic functors of the generalised species.

The central part of the proof of the theorem is encapsulated in the following lemma.

\subsubsection{Lemma.}

(i) There is an isomorphism natural for $X \in \widehat{A}$ and $u_{j} \in(\mathbf{P} A)^{\circ}$, for $j \in[n]$, as follows:

$$
\prod_{j \in[n]} X^{u_{j}} \cong X^{\oplus_{j \in[n]} u_{j}}
$$

(ii) For every $(A, B)$-species $F$ there is an isomorphism natural for $X \in \widehat{A}$ and $v \in(\mathbf{P} B)^{\circ}$ as follows:

$$
(\widetilde{F} X)^{v} \cong \int^{u \in \mathbf{P} A} F^{* v}(u) \times X^{u}
$$

Proof.

(i) $\prod_{j \in[n]} X^{u_{j}} \cong \prod_{j \in[n]} \widehat{A}\left[\mathrm{~S}_{A}\left(u_{j}\right), X\right]$

$$
\begin{aligned}
& \cong \widehat{A}\left[\sum_{j \in[n]} \mathrm{S}_{A}\left(u_{j}\right), X\right] \\
& \cong \widehat{A}\left[\mathrm{~S}_{A}\left(\oplus_{j \in[n]} u_{j}\right), X\right], \text { by }(7) \\
& \cong X^{\oplus_{j \in[n]} u_{j}}
\end{aligned}
$$


(ii) $(\widetilde{F} X)^{\left\langle b_{j}\right\rangle_{j \in[n]}}$

$$
\begin{aligned}
& =\prod_{j \in[n]} \int^{u \in \mathbf{P} A} F(u)_{b_{j}} \times X^{u} \\
& \cong \int^{u_{1}, \ldots, u_{n} \in \mathbf{P} A} \prod_{j \in[n]} F\left(u_{j}\right)_{b_{j}} \times \prod_{j \in[n]} X^{u_{j}} \\
& \cong \int^{u_{1}, \ldots, u_{n} \in \mathbf{P} A} \prod_{j \in[n]} F\left(u_{j}\right)_{b_{j}} \times X^{\oplus_{j \in[n]} u_{j}} \quad, \text { by }(11) \\
& \cong \int^{u \in \mathbf{P} A} \int^{u_{1}, \ldots, u_{n} \in \mathbf{P} A} \prod_{j \in[n]} F\left(u_{j}\right)_{b_{j}} \times \mathbf{P} A\left[\oplus_{j \in[n]} u_{j}, u\right] \times X^{u} \\
& =\int^{u \in \mathbf{P} A} F^{*\left\langle b_{j}\right\rangle_{j \in[n]}}(u) \times X^{u}
\end{aligned}
$$

Proof of Theorem 3.2.1. We establish that, for an $(A, B)$-species $F$ and a $(B, C)$-species $G$, the functorial composition of analytic functors $\widetilde{G} \widetilde{F}$ is naturally isomorphic to the analytic functor $\widetilde{G \circ F}$.

Indeed, for $X \in \widehat{A}$, we have the following natural isomorphisms:

$$
\begin{aligned}
\widetilde{G}(\widetilde{F} X)_{c} & =\int^{v \in \mathbf{P} B} G(v)_{c} \times(\widetilde{F} X)^{v} \\
& \cong \int^{v \in \mathbf{P} B} G(v)_{c} \times \int^{u \in \mathbf{P} A} F^{* v}(u) \times X^{u} \quad \text { by (12) } \\
& \cong \int^{u \in \mathbf{P} A} \int^{v \in \mathbf{P} B} G(v)_{c} \times F^{* v}(u) \times X^{u} \\
& =\int^{u \in \mathbf{P} A}(G \circ F)(u)_{c} \times X^{u} \\
& =\widetilde{G \circ F}(X)_{c}
\end{aligned}
$$

One can also generalise the definition of the unit of the tensor product of the substitution monoidal structure recalled in (5). This is done by defining the identity $(A, A)$-species of structures $I_{A}$ as

$$
I_{A}(a, u)=_{\operatorname{def}} \mathbf{P} A[\langle a\rangle, u] .
$$

As expected, the identities generalised species are characterised by the fact that $\widetilde{I_{A}} \cong \operatorname{Id}_{\widehat{A}}$.

3.3. The bicategory of generalised species. There is a bicategory Esp (for espèces de structures [23, 24]) with small categories as 0-cells, generalised species of structures as 1-cells, and natural transformations as 2-cells. Modulo the equivalence (6), composition of 1-cells in Esp is given by the substitution operation defined in (9) and the identity 1-cells are given by the identity generalised species defined in (13). Indeed, it is possible to exhibit coherent natural isomorphisms

$$
I_{B} \circ F \stackrel{\cong}{\longrightarrow} F, \quad F \stackrel{\cong}{\longrightarrow} F \circ I_{A}, \quad(H \circ G) \circ F \stackrel{\cong}{\rightarrow} H \circ(G \circ F)
$$


that arise from lengthy coend manipulations. The next section describes a conceptual approach to the formulation of the substitution calculus of generalised species of structures.

\section{The Kleisli Bicategory of generalised species}

4.1. The 2-monad for symmetric monoidal categories. The function mapping a category $A$ to the category $\mathbf{P} A$ defines the action on objects of a 2-endofunctor which is part of a 2-monad $\mathrm{P}$ on the 2-category CAT of locally small categories, functors, and natural transformations [8]. As with any 2-monad, we have both strict algebras and pseudo-algebras [43]. The strict algebras and the pseudo-algebras for $\mathrm{P}$ are categories equipped with particular forms of symmetric monoidal structure. The strict algebras are symmetric strict monoidal categories, and $\mathrm{P} A$ is the free symmetric strict monoidal category on $A$. The pseudo-algebras are instead symmetric monoidal categories in the unbiased version (see, e.g., [32, Section 3.1]). These are categories equipped with coherent $n$-ary symmetric tensor products for all $n \in \mathbb{N}$, instead of just nullary and binary ones.

We describe the unit and multiplication of the 2 -monad P. The unit $e: \mathrm{Id} \longrightarrow \mathrm{P}$ has components given by the embeddings $e_{A}: A \hookrightarrow \mathrm{P} A$ defined as

$$
e_{A}(a)={ }_{\text {def }}\langle a\rangle .
$$

The components $m_{A}: \mathrm{P}^{2} A \rightarrow \mathrm{P} A$ of the multiplication $m: \mathrm{P}^{2} \rightarrow \mathrm{P}$ are defined using the canonical tensor product on $\mathrm{P} A$ defined in (8) by letting

$$
m_{A}\left(\left\langle u_{i}\right\rangle_{i \in[n]}\right)=_{\text {def }} \bigoplus_{i \in[n]} u_{i} .
$$

As it is to be expected in the context of 2-dimensional monad theory [8], the universal property of $\mathrm{P} A$ requires that for any functor $F: A \rightarrow B$, where $B$ is a (unbiased) symmetric monoidal category, we have a diagram of the form

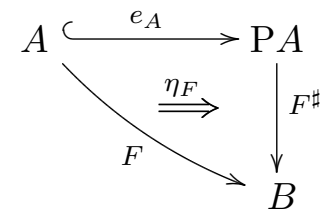

where $F^{\sharp}$ is a symmetric strong monoidal functor and $\eta_{F}$ is a natural isomorphism. If $B$ is strict monoidal, then $F^{\sharp}$ is the unique symmetric strict monoidal functor that makes diagram (14) commute strictly, rather than up to isomorphism. These data are universal in that they induce an equivalence of categories

$$
\operatorname{CAT}[A, B] \underset{\frac{(-)^{\sharp}}{\simeq}}{\simeq} \operatorname{SMON}[\mathrm{P} A, B]
$$


where SMON denotes the 2-category of (unbiased) symmetric monoidal locally small categories, symmetric strong monoidal functors, and monoidal transformations; $U: \mathbf{S M O N} \rightarrow \mathbf{C A T}$ is the forgetful 2-functor.

The functor $F^{\sharp}: \mathrm{P} A \rightarrow B$ can be defined explicitly using the $n$-ary tensor product of $B$ as follows:

$$
F^{\sharp}\left(\left\langle a_{i}\right\rangle_{i \in[n]}\right)={ }_{\text {def }} \bigotimes_{i \in[n]} F\left(a_{i}\right) .
$$

Similarly, the natural isomorphism $\eta_{F}$ can be defined using the coherence isomorphisms of the (unbiased) symmetric monoidal structure of $B$.

4.2. A pseudo-monad on profunctors. Recall that for small categories $A$ and $B$, an $(A, B)$-profunctor $A+B$ is a functor $A \rightarrow \widehat{B}$, or equivalently a functor $B^{\circ} \times A \rightarrow$ Set. The identity profunctor on $A$ is the Yoneda embedding $\mathrm{y}_{A}: A \hookrightarrow \widehat{A}$, corresponding to the hom functor $A^{\circ} \times A \rightarrow$ Set. The composite of two profunctors $F: A \nrightarrow B$ and $G: B \nrightarrow C$, denoted $G \cdot F: A \nrightarrow C$, is defined as follows:

$$
(G \cdot F)(c, a)={ }_{\operatorname{def}} \int^{b \in B} G(c, b) \times F(b, a) .
$$

We write Prof for the bicategory of small categories, profunctors, and natural transformations; see e.g. $[4,31,44]$ for further background.

The possibility of extending the 2-monad for symmetric [strict] monoidal small categories to Prof is an example of a general situation analysed in detail in the context of 2-dimensional monad theory in [18], which develops further the theory of pseudo-distributive laws for pseudo-monads [26, 34, 11, 19]. In the particular case that concerns us here, one can see that the key reason for this phenomenon is that the (symmetric) monoidal structure on a category extends to its category of presheaves via Day's convolution monoidal structure $[12,22]$. Recall that for a small category $B$, the convolution tensor product of presheaves $X_{i} \in \widehat{\mathrm{PB}}$, for $i \in[n]$, is the presheaf defined as

$$
\left(\widehat{\bigotimes}_{i \in[n]} X_{i}\right)(v)=_{\operatorname{def}} \int^{v_{1}, \ldots, v_{n} \in \mathrm{P} B} \prod_{i \in[n]} X_{i}\left(v_{i}\right) \times \mathrm{P} B\left[v, \oplus_{i \in[n]} v_{i}\right] .
$$

As a consequence, for a small category $B$, the cocompleteness and the (unbiased) monoidal structure of $\widehat{\mathrm{PB}}$ allow us to build the following diagram

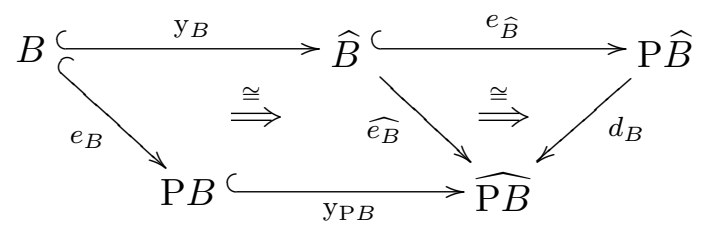

Here $\widehat{e_{B}}$ is the left Kan extension of the composite $\mathrm{y}_{\mathrm{P}} e_{B}$ along the Yoneda embedding given by

$$
\left(\widehat{e_{B}} X\right)(v)=_{\operatorname{def}} \int^{b \in B} X(b) \times \mathrm{P} B\left[v, e_{B}(b)\right],
$$


and $d_{B}={ }_{\text {def }}{\widehat{e_{B}}}^{\sharp}$ according to the notation introduced in (15). Using this construction, for a profunctor $F: A \mapsto B$, one can define a profunctor $\overline{\mathrm{P}} F: \mathrm{P} A \longrightarrow \mathrm{P} B$ as the composite

$$
\mathrm{P} A \stackrel{\mathrm{P} F}{\longrightarrow} \mathrm{P} \widehat{B} \stackrel{d_{B}}{\longrightarrow} \widehat{\mathrm{PB}}
$$

This definition yields a pseudo-endofunctor $\overline{\mathrm{P}}$ on Prof which underlies a pseudo-monad. The unit and multiplication of this pseudo-monad are defined using the unit and multiplication of the 2-monad $\mathrm{P}$. The components of the unit are the profunctors $\bar{e}_{A}: A \longrightarrow \mathrm{P} A$ defined as

$$
\bar{e}_{A}(u, a)={ }_{\operatorname{def}} \mathrm{P} A\left[u, e_{A}(a)\right] .
$$

The multiplication has components $\bar{m}_{A}: \mathrm{P}^{2} A \nrightarrow \mathrm{P} A$ given by profunctors defined as

$$
\bar{m}_{A}(u, s)=_{\text {def }} \mathrm{P} A\left[u, m_{A}(s)\right] .
$$

4.3. The Kleisli bicategory of generalised species. The duality on profunctors (see, e.g., [13, Section 7]) allows then to turn the pseudo-monad $\overline{\mathrm{P}}$ into a pseudo-comonad $\underline{\mathrm{P}}$.

The dual of a small category $A$ is its opposite, $A^{\perp}={ }_{\text {def }} A^{\circ}$; the dual of a profunctor $F: A \rightarrow B$ is the profunctor $F^{\perp}: B^{\perp} \nrightarrow \rightarrow A^{\perp}$ defined by letting $F^{\perp}(a, b)=_{\text {def }} F(b, a)$; the dual of a natural transformation $\eta$ : $F \Longrightarrow G: A \longrightarrow B$ is the natural transformation $\eta^{\perp}: F^{\perp} \Longrightarrow G^{\perp}: A^{\perp} \longrightarrow B^{\perp}$ given by $\left(\eta^{\perp}\right)_{(b, a)}=\eta_{(a, b)}$. Thus, the pseudo-endofunctor underlying the pseudo-comonad $\underline{\mathrm{P}}$ is given by the composite

$$
\text { Prof } \stackrel{(\cdot)^{\perp}}{\longrightarrow} \text { Prof } \stackrel{\overline{\mathrm{P}}}{\longrightarrow} \text { Prof } \stackrel{(\cdot)^{\perp}}{\longrightarrow} \text { Prof . }
$$

The counit $\underline{e}: \underline{\mathrm{P}} \longrightarrow \mathrm{Id}$ and comultiplication $\underline{m}: \underline{\mathrm{P}}^{2} \longrightarrow \underline{\mathrm{P}}$ are defined as follows

$$
\underline{e}_{A}={ }_{\text {def }}\left(\bar{e}_{A^{\perp}}\right)^{\perp}, \quad \underline{m}_{A}={ }_{\operatorname{def}}\left(\bar{m}_{A^{\perp}}\right)^{\perp} .
$$

We write Prof P $_{\mathrm{P}}$ for the Kleisli bicategory of the pseudo-comonad $\underline{\mathrm{P}}$ on Prof. The general Kleisli-bicategory construction is recalled in Appendix A.6. For our particular case, the 0-cells are small categories and, since $\operatorname{Prof}_{\mathrm{P}}[A, B]=\operatorname{Prof}[\mathrm{P} A, B]$, the 1-cells are generalised species of structures. Henceforth, when considered as a 1-cell in $\operatorname{Prof}_{\underline{P}}$, an $(A, B)$-species $F$ will be denoted in either of the following ways

$$
F: \mathrm{P} A \rightarrow \widehat{B}, \quad F: \mathrm{P} A \nrightarrow B, \quad F: A \longleftrightarrow B
$$

depending on what is most convenient.

The identities in the Kleisli bicategory of generalised species Prof $\mathbf{P}_{\underline{P}}$, which are given by the components of the counit of $\underline{P}$, coincide with the identities generalised species defined in (13). There is also a correspondence between composition and substitution.

4.3.1. Theorem. The composition and substitution operations of generalised species of structures are naturally isomorphic. 
It is convenient for what follows to adopt the following convention. Since the opposite of a (symmetric) [strict] monoidal category is also (symmetric) [strict] monoidal, for any small category $A$, the category $(\mathrm{P} A)^{\circ}$ is symmetric strict monoidal, and one can consider the symmetric monoidal extension of the embedding $e_{A}{ }^{\circ}: A^{\circ} \hookrightarrow(\mathrm{P} A)^{\circ}$ along the universal embedding $e_{A^{\circ}}$ : $A^{\circ} \hookrightarrow \mathrm{P}\left(A^{\circ}\right)$. This construction yields a canonical natural isomorphism

$$
\left(e_{A}^{\circ}\right)^{\sharp}: \mathrm{P}\left(A^{\circ}\right) \stackrel{\cong}{\longrightarrow}(\mathrm{P} A)^{\circ}
$$

which, as a notational convention, we will consistently treat as an identity. Conveniently then, the expression $\mathrm{P} A^{\circ}$ and similar ones become unambiguous.

Proof of Theorem 4.3.1. Let $F: A \leftrightarrow B$ and $G: B \leftrightarrow C$ be generalised species. Recall from Appendix A.6 that the Kleisli composite of $F$ and $G$ is the profunctor composite $G \cdot F^{\mathrm{P}}: \mathrm{P} A \longrightarrow C$ where $F^{\mathrm{P}}: \mathrm{P} A \longrightarrow \mathrm{P} B$ is in turn the profunctor composite

$$
\underline{\mathrm{P}}(F) \cdot \underline{m}_{A}=\left(\overline{\mathrm{P}}\left(F^{\perp}\right)\right)^{\perp} \cdot \underline{m}_{A} .
$$

Thus, it is enough to show that $F \stackrel{\mathrm{P}}{\mathrm{P}} \mathrm{P} A \nrightarrow \mathrm{P} B$ is naturally isomorphic to $F^{*}: \mathrm{P} A \rightarrow \mathrm{P} B$ defined, using (10), as follows

$$
F^{*}(v, u)=_{\text {def }} F^{* v}(u) .
$$

We start by considering $\overline{\mathrm{P}}\left(F^{\perp}\right): \mathrm{P} B^{\circ} \rightarrow \widehat{\mathrm{P}^{2} A^{\circ}}$. For $\left\langle b_{j}\right\rangle_{j \in[n]} \in \mathrm{P} B^{\circ}$, we have that

$$
\begin{aligned}
\overline{\mathrm{P}}\left(F^{\perp}\right)\left(\left\langle b_{j}\right\rangle_{j \in[n]}\right) & =d_{\mathrm{P} A^{\circ}}\left(\mathrm{P}\left(F^{\perp}\right)\left(\left\langle b_{j}\right\rangle_{j \in[n]}\right)\right) \\
& =\widehat{e_{\mathrm{P} A^{\circ}}} \sharp\left(\left\langle F^{\perp}\left(b_{j}\right)\right\rangle_{j \in[n]}\right) \\
& =\widehat{\oplus}_{j \in[n]} \widehat{e_{\mathrm{P} A^{\circ}}}\left(F^{\perp}\left(b_{j}\right)\right)
\end{aligned}
$$

Hence, for $s \in \mathrm{P}^{2} A$, we have

$\overline{\mathrm{P}}\left(F^{\perp}\right)\left(s,\left\langle b_{j}\right\rangle_{j \in[n]}\right)=\int^{s_{1}, \ldots, s_{n} \in \mathrm{P}^{2} A} \prod_{j \in[n]} \widehat{\mathrm{PA}^{\circ}}\left(F^{\perp}\left(b_{j}\right)\right)\left(s_{j}\right) \times \mathrm{P}^{2} A\left[\oplus_{j \in[n]} s_{j}, s\right]$.

For $b \in \mathrm{P} B^{\circ}$ and $s \in \mathrm{P}^{2} A$, we have

$$
\widehat{e_{\mathrm{PA}}^{\circ}}\left(F^{\perp}(b)\right)(s)=\int^{u \in \mathrm{P} A} F(b, u) \times \mathrm{P}^{2} A[\langle u\rangle, s] .
$$

It follows that, for $\left\langle b_{j}\right\rangle_{j \in[n]} \in \mathrm{P} B^{\circ}$ and $s \in \mathrm{P}^{2} A$,

$$
\begin{aligned}
& (\underline{\mathrm{P}} F)\left(\left\langle b_{j}\right\rangle_{j \in[n]}, s\right) \\
& \quad=\overline{\mathrm{P}}\left(F^{\perp}\right)\left(s,\left\langle b_{j}\right\rangle_{j \in[n]}\right) \\
& \quad=\int^{s_{1}, \ldots, s_{n} \in \mathrm{P}^{2} A} \prod_{j \in[n]} \int^{u_{j} \in \mathrm{P} A} F\left(b_{j}, u_{j}\right) \times \mathrm{P}^{2} A\left[\left\langle u_{j}\right\rangle, s_{j}\right] \times \mathrm{P}^{2} A\left[\oplus_{j \in[n]} s_{j}, s\right] \\
& \quad \cong \int^{u_{1}, \ldots, u_{n} \in \mathrm{P} A} \prod_{j \in[n]} F\left(b_{j}, u_{j}\right) \times \mathrm{P}^{2} A\left[\left\langle u_{j}\right\rangle_{j \in[n]}, s\right]
\end{aligned}
$$


Finally, for $\left\langle b_{j}\right\rangle_{j \in[n]} \in \mathrm{P} B^{\circ}$ and $u \in \mathrm{P} A$, we conclude that

$$
\begin{aligned}
F & \underline{\mathrm{P}}\left(\left\langle b_{j}\right\rangle_{j \in[n]}, u\right) \\
& =\iint^{s \in \mathrm{P}^{2} A}(\underline{\mathrm{P}} F)\left(\left\langle b_{j}\right\rangle_{j \in[n]}, s\right) \times \mathrm{P} A\left[m_{A}(s), u\right] \\
& \cong \int^{s \in \mathrm{P}^{2} A} \int^{u_{1}, \ldots, u_{n} \in \mathrm{P} A} \prod_{j \in[n]} F\left(b_{j}, u_{j}\right) \times \mathrm{P}^{2} A\left[\left\langle u_{j}\right\rangle_{j \in[n]}, s\right] \times \mathrm{P} A\left[m_{A}(s), u\right] \\
& \cong \int^{u_{1}, \ldots, u_{n} \in \mathrm{P} A} \prod_{j \in[n]} F\left(b_{j}, u_{j}\right) \times \mathrm{P} A\left[\oplus_{j \in[n]} u_{j}, u\right] \\
& =F^{*}\left(\left\langle b_{j}\right\rangle_{j \in[n]}, u\right)
\end{aligned}
$$

The correspondence between substitution and composition extends to a biequivalence between Esp and Prof $_{\bar{P}}$.

\section{The Cartesian Closed structure}

The concept of cartesian closed bicategory can be expressed entirely in terms of the concept of biadjunction between bicategories [44], the definition of which we recall in Appendix A.4.

A bicategory $\mathcal{E}$ is cartesian if the diagonal pseudo-functor $\Delta_{n}: \mathcal{E} \rightarrow \mathcal{E}^{n}$ has a right biadjoint for all $n \in \mathbb{N}$. Further, a bicategory $\mathcal{E}$ with binary products $(-) \sqcap(=): \mathcal{E} \times \mathcal{E} \rightarrow \mathcal{E}$ is closed if, for all $B \in \mathcal{E}$, the pseudofunctor $(-) \sqcap B: \mathcal{E} \rightarrow \mathcal{E}$ has a right biadjoint.

5.1. Products. To establish the cartesian structure of the bicategory of generalised species, we first recall a basic property of the presheaf construction, viz. that it maps sums to products.

Let $n \in \mathbb{N}$. For presheaves $X_{i}: A_{i}{ }^{\circ} \rightarrow$ Set $(i \in[n])$, let $\left[X_{1}, \ldots, X_{n}\right]$ : $\left(\sum_{i \in[n]} A_{i}\right)^{\circ} \rightarrow$ Set be the unique presheaf such that for all $i \in[n]$, $\left[X_{1}, \ldots, X_{n}\right] \iota_{i}{ }^{\circ}=X_{i}$, where $\iota_{i}: A_{i} \rightarrow \sum_{i \in[n]} A_{i}$ denote the coproduct injections. This construction yields a functor $[-]: \prod_{i \in[n]} \widehat{A_{i}} \rightarrow \widehat{\sum_{i \in[n]}} A_{i}$. Conversely, composition with the coproduct injections provides a functor in the opposite direction, $\iota: \widehat{\sum_{i \in[n]}} A_{i} \rightarrow \prod_{i \in[n]} \widehat{A}_{i}$. This is defined by letting $\iota(F)={ }_{\text {def }}\left(F \iota_{1}^{\circ}, \ldots, F \iota_{n}^{\circ}\right)$. We then have the following fact.

\subsubsection{Lemma. The functors}

$$
\prod_{i \in[n]} \widehat{A_{i}} \underset{\iota}{\longleftarrow} \widehat{\sum_{i \in[n]}} A_{i}
$$

form an isomorphism of categories. 
This lemma entails the following chain of isomorphisms

$$
\begin{aligned}
\prod_{i \in[n]} \operatorname{Esp}\left[B, A_{i}\right] & =\prod_{i \in[n]}\left[\mathrm{P} B, \widehat{A_{i}}\right] \\
& \cong\left[\mathrm{P} B, \prod_{i \in[n]} \widehat{A_{i}}\right] \\
& \cong\left[\mathrm{P} B, \widehat{\sum_{i \in[n]}} A_{i}\right] \\
& =\operatorname{Esp}\left[B, \sum_{i \in[n]} A_{i}\right]
\end{aligned}
$$

which indicate that the bicategory of generalised species has finite products. The next result establishes this formally.

\subsubsection{Theorem. The bicategory Esp is cartesian.}

Proof. We start by defining the action of the right biadjoint to the diagonal pseudo-functor on objects. For small categories $A_{i}(i \in[n])$,

$$
\prod_{i \in[n]} A_{i}=_{\operatorname{def}} \sum_{i \in[n]} A_{i} .
$$

Then, for $G_{i}: B \longrightarrow A_{i}(i \in[n])$, the pairing $\left\langle G_{1}, \ldots, G_{n}\right\rangle: B \imath \rightarrow \prod_{i \in[n]} A_{i}$ is defined by setting, for $i \in[n]$,

$$
\left\langle G_{1}, \ldots, G_{n}\right\rangle\left(\iota_{i}(a), v\right)=_{\text {def }} G_{i}(a, v)
$$

where $a \in A_{i}$. Next, we introduce the projections $\pi_{i}: \prod_{i \in[n]} A_{i} \longleftrightarrow A_{i}(i \in[n])$,

$$
\pi_{i}(a, u)=_{\operatorname{def}} \mathrm{P}\left(\sum_{i \in[n]} A_{i}\right)\left[\left\langle\iota_{i}(a)\right\rangle, u\right] .
$$

We claim that these data determine an adjoint equivalence of the form

$$
\operatorname{Esp}\left[B, \prod_{i \in[n]} A_{i}\right] \underset{<}{\stackrel{\left(\pi_{1} \circ(-), \ldots, \pi_{n} \circ(-)\right)}{\perp}} \prod_{i \in[n]} \operatorname{Esp}\left[B, A_{i}\right] .
$$

To check this, one needs to exhibit natural isomorphisms for the unit and counit. The component of the unit for $F: B \leftrightarrow \prod_{i \in[n]} A_{i}$ is given by a natural isomorphism

$$
F \stackrel{\cong}{\Longrightarrow}\left\langle\pi_{1} \circ F, \ldots, \pi_{n} \circ F\right\rangle
$$

that expresses the $\eta$-expansion for products. The component of the counit for $G_{i}: B \longrightarrow A_{i}(i \in[n])$, consists of natural isomorphisms

$$
\pi_{i} \circ\left\langle G_{1}, \ldots, G_{n}\right\rangle \stackrel{\cong}{\Longrightarrow} G_{i}
$$

that express the $\beta$-reduction for products. Explicit definitions can be derived via coend manipulations which we omit. 
5.2. Exponentials. The closed structure of the cartesian bicategory of generalised species will be established by exploiting a property of the free symmetric strict monoidal completion. Since the product of (symmetric) [strict] monoidal categories carries a (symmetric) [strict] monoidal structure, we get a diagram of the form

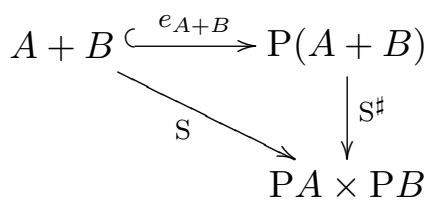

where $\mathrm{S}: A+B \rightarrow \mathrm{P} A \times \mathrm{P} B$ is defined by letting

$$
\mathrm{S}\left(\iota_{1}(a)\right)=_{\text {def }}(\langle a\rangle,\langle\rangle), \quad \mathrm{S}\left(\iota_{2}(b)\right)=_{\text {def }}(\langle\rangle,\langle b\rangle)
$$

for $a \in A$ and $b \in B$. We also define a functor in the opposite direction:

$$
\mathrm{P} A \times \mathrm{P} B \stackrel{\mathrm{P}\left(\iota_{1}\right) \times \mathrm{P}\left(\iota_{2}\right)}{\longrightarrow} \mathrm{P}(A+B) \times \mathrm{P}(A+B) \stackrel{\oplus}{\longrightarrow} \mathrm{P}(A+B) .
$$

5.2.1. Lemma. The functors

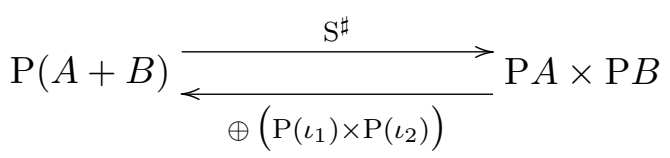

form an equivalence of categories.

We introduce some abbreviations for future reference. For $x \in \mathrm{P}(A \times B)$, we write $\left(x_{\cdot_{1}}, x_{\cdot_{2}}\right)$ for $\mathrm{S}^{\sharp}(x)$, so that we have $x_{\cdot_{1}} \in \mathrm{P} A$ and $x_{\cdot_{2}} \in \mathrm{P} B$. Given $u \in \mathrm{P} A$ and $v \in \mathrm{P} B$, we write $u \oplus v$ for $\mathrm{P} \iota_{1}(u) \oplus \mathrm{P} \iota_{2}(v)$, so that we have $u \oplus v \in \mathrm{P}(A+B)$. With this notation, thus, we then have isomorphisms $x \cong x_{\cdot 1} \oplus x_{\cdot 2}$, for all $x \in \mathrm{P}(A+B)$.

Crucially, the equivalence of Lemma 5.2.1 can be used in the following chain of equivalences, that suggests the definition of exponentials in the bicategory of generalised species of structures:

$$
\begin{aligned}
\operatorname{Esp}[A \sqcap B, C] & =[\mathrm{P}(A+B), \widehat{C}] \\
& \simeq[\mathrm{P} A \times \mathrm{P} B, \widehat{C}] \\
& \cong\left[\mathrm{P} A, \mathrm{P} \widehat{B^{\circ} \times} C\right] \\
& =\operatorname{Esp}\left[A, \mathrm{P} B^{\circ} \times C\right]
\end{aligned}
$$

In the proof of the next theorem, the cartesian closed structure is exhibited in detail.

5.2.2. Theorem. The cartesian bicategory Esp is closed.

Proof. Let $B \in \mathbf{E s p}$ and recall that the pseudo-functor $(-) \sqcap B:$ Esp $\rightarrow$ Esp is defined using the projections and pairing operations introduced earlier, as follows:

$$
F \sqcap B=_{\text {def }}\left\langle F \circ \pi_{1}, \pi_{2}\right\rangle .
$$


To define the required right biadjoint we let, for $C \in \mathbf{E s p}$,

$$
C^{B}={ }_{\text {def }} \mathrm{P} B^{\circ} \times C .
$$

For $G: A \sqcap B \longleftrightarrow C$, we define its abstraction $\lambda_{B}(G): A \longleftrightarrow C^{B}$ as follows

$$
\left(\lambda_{B} G\right)((v, c), u)=_{\operatorname{def}} G(c, u \oplus v) .
$$

We further define the generalised species eval $_{B}: C^{B} \sqcap B \longmapsto C$, which models evaluation, as follows

$$
\operatorname{eval}_{B}(c, z)=_{\text {def }} \mathrm{P}\left(\mathrm{P} B^{\circ} \times C\right)[\langle(z \cdot 2, c)\rangle, z \cdot 1] .
$$

We claim that there is an adjoint equivalence of the form

$$
\operatorname{Esp}\left[A, C^{B}\right] \underset{\frac{\operatorname{eval}_{B} \circ(-\sqcap B)}{\lambda_{B}(-)}}{\stackrel{\perp}{L}} \operatorname{Esp}[A \sqcap B, C] .
$$

The component of the unit of the adjunction for $F: A \leftrightarrow C^{B}$ is given by a natural isomorphism of the form

$$
F \stackrel{\cong}{=} \lambda_{B}\left(\text { eval }_{B} \circ(F \sqcap B)\right)
$$

that expresses the $\eta$-expansion for exponentials. The component of the counit for $G: A \sqcap B \rightarrow C$ is given by a natural isomorphism of the form

$$
\operatorname{eval}_{B} \circ\left(\lambda_{B}(G) \sqcap B\right) \stackrel{\cong}{=} G
$$

that expresses the $\beta$-reduction for exponentials. These natural transformations can be produced by lengthy coend manipulations.

\section{Applications}

6.1. Structural combinatorics. The concept of a generalised species of structures encompasses most of the notions of combinatorial species introduced in the literature. Let us refer here to $(A, \mathbf{1})$-species of structures simply as $A$-species, and observe that these can be identified with functors $\mathbf{B} A \rightarrow$ Set. Under these conventions, one can see that $k$-sorted species [23, 7] are $\left(\coprod_{i \in[k]} \mathbf{1}\right)$-species; permutationals [6, 23] are $\mathbf{C P}$-species, where $\mathbf{C P}$ is the groupoid of finite cyclic permutations; partitionals [40] are $\mathbf{B}^{*}$-species, where $\mathbf{B}^{*}$ is the groupoid of non-empty finite sets. Other notions fitting into our framework are colored species [38], and species on graphs and digraphs [37].

Furthermore, the operation of substitution associated to these combinatorial species (introduced for enumerative combinatorial purposes as structural counterparts of the composition of corresponding formal power series) appear as specialisations of our substitution operation for generalised species. For example, the multi-substitution $G\left(F_{1}, \ldots, F_{k}\right)$ of the $\ell$-sorted species $F_{1}, \ldots, F_{k}$ and the $k$-sorted species $G$ is the substitution $\ell$-sorted species $G \circ\left\langle F_{1}, \ldots, F_{k}\right\rangle$. 
In fact, the whole calculus of species [24, 7] (encompassing addition, multiplication, substitution, [partial] differentiation) extends to a calculus of generalised species. However, the closed structure exhibited in this paper provides a new dimension absent from the combinatorial perspective. Indeed, for instance, the calculus of generalised species does not only supports the above operations but also higher-order differential operators. Details will appear elsewhere, though see [17, Section 2].

6.2. Lambda calculus. The bicategory of generalised species, being cartesian closed, is a pseudo-extensional model of the simply typed lambda calculus, in the sense that both the $\eta$ and $\beta$ identities for product and function types (see, e.g., [30]) are modelled by canonical natural isomorphisms rather than by equalities. Note that our explicit description of the cartesian closed structure includes that of the $\eta$ and $\beta$ isomorphisms, respectively modelling $\eta$-expansion and $\beta$-reduction.

The bicategory of generalised species further provides models of the untyped lambda calculus (i.e. reflexive objects [42]) akin to graph models (see, e.g., $[3,29]$ and also [21]). Indeed, the free $\mathrm{P}(-)^{\circ} \times(-)$-algebra $\bar{A}$ on a small category $A$ in Cat, the category of small categories and functors, yields a retraction $\bar{A}^{\bar{A}} \triangleleft \bar{A}$ in Esp. Further, the final $\mathrm{P}(-)^{\circ} \times(-)$-coalgebra $\mathbf{U}$ on Cat yields an isomorphism $\mathbf{U}^{\mathbf{U}} \cong \mathbf{U}$ in Esp. Curiously, $\mathbf{U}$ is a groupoid with the following explicit combinatorial description: it has objects given by the class of planar trees described by $\omega^{\circ}$-chains

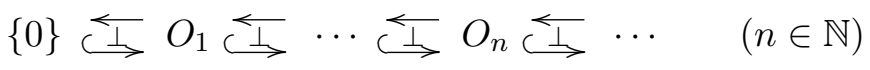

of reflections between finite ordinals, with morphisms given by natural bijections. In the light of the differential structure mentioned in Section 6.1, the bicategory of generalised species provides also a 2-dimensional model of both the typed and the untyped differential lambda calculus $[15,9]$.

Acknowledgements. We are grateful to Claudio Hermida for advice on bicategory theory.

\section{ApPendix A. BicAtegory THEORY}

A.1. Bicategories. A bicategory $\mathcal{C}$ consists of the following data:

- a class $O b(\mathcal{C})$ of 0 -cells, or objects;

- a family $\mathcal{C}[A, B]$, for $A, B \in O b(\mathcal{C})$, of hom-categories, whose objects and morphisms are respectively called 1-cells and 2-cells;

- a composition operation, given by a family of functors

$$
\mathcal{C}[B, C] \times \mathcal{C}[A, B] \longrightarrow \mathcal{C}[A, C]
$$

whose actions on a pair $(G, F)$ of 1 -cells is written $G \cdot F$;

- identities, given by 1 -cells $1_{A} \in \mathcal{C}[A, A]$, for $A \in O b(\mathcal{C})$; 
- natural isomorphisms $\alpha, \lambda, \rho$, expressing the associativity law

$$
\alpha_{H, G, F}:(H \cdot G) \cdot F \rightarrow H \cdot(G \cdot F)
$$

and identity laws

$$
\lambda_{F}: 1_{B} \cdot f \longrightarrow F, \quad \rho_{F}: F \rightarrow F \cdot 1_{A}
$$

subject to three coherence axioms [5].

A.2. Pseudo-functors. A pseudo-functor $\Phi: \mathcal{C} \rightarrow \mathcal{D}$ between bicategories consists of

- a function $\Phi: O b(\mathcal{C}) \rightarrow O b(\mathcal{D})$;

- functors $\Phi_{A, B}: \mathcal{C}[A, B] \rightarrow \mathcal{D}[\Phi A, \Phi B]$, for $A, B \in \mathcal{C}$;

- natural isomorphisms

$$
\varphi_{G, F}: \Phi(G \cdot F) \rightarrow \Phi(G) \cdot \Phi(F), \quad \varphi_{A}: \Phi\left(1_{A}\right) \rightarrow 1_{\Phi A}
$$

subject to coherence axioms [5].

A.3. Pseudo-natural transformations. A pseudo-natural transformation $p: \Phi \longrightarrow \Psi$ between pseudo-functors consists of

- a family of morphisms $p_{A}: \Phi A \rightarrow \Psi A$, for $A \in \mathcal{C}$;

- a family of invertible 2-cells

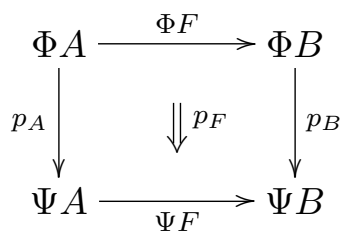

for $F: A \rightarrow B$, subject to coherence axioms [5].

A.4. Biadjoints. To define a right biadjoint to a pseudo-functor between bicategories $\Phi: \mathcal{C} \longrightarrow \mathcal{D}$, it is sufficient to give:

- 0-cells $\Psi X \in \mathcal{C}$, for $X \in \mathcal{D}$;

- 1-cells $q_{X}: \Phi \Psi X \rightarrow X$, for $X \in \mathcal{D}$;

- a family of equivalences of categories, for $A \in \mathcal{C}$ and $X \in \mathcal{D}$,

$$
\mathcal{C}[A, \Psi X] \underset{\frac{q_{X} \cdot \Phi(\cdot)}{(\cdot)^{b}}}{\frac{\perp}{\longleftrightarrow}} \mathcal{D}[\Phi A, X] .
$$

The unit and counit of an adjunction as in (16) have components that we write, for $F: A \longrightarrow \Psi X$ and $G: \Phi A \longrightarrow X$, as

$$
\eta_{F}: F \rightarrow\left(q_{X} \cdot \Phi(F)\right)^{b}, \quad \varepsilon_{G}: q_{X} \cdot \Phi\left(G^{b}\right) \rightarrow G .
$$

These data canonically determine a pseudo-functor $\Psi: \mathcal{D} \rightarrow \mathcal{C}$ and the unit and counit of the pseudo-adjunction, given by pseudo-natural transformations $p: \operatorname{Id}_{\mathcal{C}} \longrightarrow \Psi \Phi$ and $q: \Phi \Psi \rightarrow \operatorname{Id}_{\mathcal{D}}$, which satisfy the triangular laws up to coherent isomorphism. 
A.5. Pseudo-comonads. A pseudo-comonad on a bicategory $\mathcal{C}$ consists of a pseudo-functor $P: \mathcal{C} \rightarrow \mathcal{C}$, two pseudo-natural transformations $v: P \rightarrow \operatorname{Id}_{\mathcal{C}}$ and $n: P \longrightarrow P^{2}$, called counit and comultiplication, and invertible modifications $\alpha, \lambda$, and $\rho$ fitting in the following diagrams
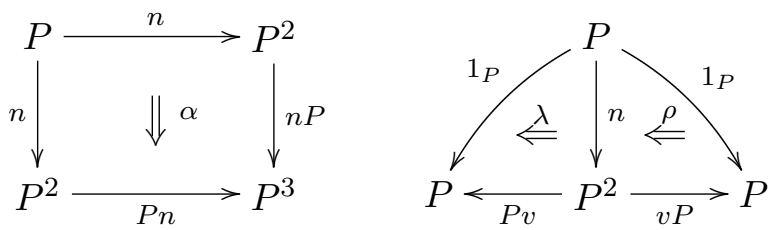

and subject to three coherence conditions. We refer to the modifications $\alpha$, $\lambda$, and $\rho$ as the associativity, left unit, and right unit of the pseudo-monad, respectively.

A.6. The Kleisli bicategory. The Kleisli bicategory $\mathcal{C}_{P}$ associated to a pseudo-comonad $P$ on a bicategory $\mathcal{C}$ is defined as having the same 0 -cells as $\mathcal{C}$, and by letting

$$
\mathcal{C}_{P}[A, B]={ }_{\text {def }} \mathcal{C}[P A, B] .
$$

The composition in $\mathcal{C}_{P}$ of $F: P A \rightarrow B$ and $G: P B \rightarrow C$ is as

$$
G \circ F={ }_{\operatorname{def}} G \cdot\left(P F \cdot n_{A}\right)
$$

This definition can be easily extended to provide the required composition functors. The identities in $\mathcal{C}_{P}$ are the components of the counit of the comonad. The associativity, left unit, and right unit of the pseudo-comonad allow the definition of isomorphisms for the associativity and unit laws of the bicategory. The coherence conditions for a pseudo-comonad guarantee that the coherence conditions for a bicategory are satisfied.

\section{REFERENCES}

1. J. Baez and J. Dolan, Higher-dimensional algebra III: $n$-categories and the algebra of opetopes, Advances in Mathematics 135 (1998), 145-206.

2. _ From finite sets to Feynman diagrams, Mathematics Unlimited - 2001 and Beyond (B. Engquist and W. Schmid, eds.), vol. 1, Springer-Verlag, 2001, pp. 29-50.

3. H. Barendregt, The lambda calculus: Its syntax and semantics, Studies in Logic and the foundations of mathematics, vol. 103, Elsevier, 1984.

4. J. Bénabou, Distributors at work, Lecture notes by T. Streicher of a course given at TU Darmstadt, 2000. Available from $\langle$ http://www.mathematik.tu-darmstadt. de/ $\sim$ streicher $/\rangle$.

5. _ Introduction to bicategories, Reports of the Midwest Category Seminar, Lecture Notes in Mathematics, vol. 47, Springer-Verlag, 1967, pp. 1-77.

6. F. Bergeron, Une combinatoire du pléthysme, Journal of Combinatorial Theory (Series A) 46 (1987), 291-305.

7. F. Bergeron, G. Labelle, and P. Leroux, Combinatorial species and tree-like structures, Cambridge University Press, 1998.

8. R. Blackwell, G. M. Kelly, and A. J. Power, Two-dimensional monad theory, Journal of Pure and Applied Algebra 59 (1989), 1-41.

9. R. F. Blute, J. R. B. Cockett, and R. A. G. Seely, Differential categories, Mathematical Structures in Computer Science, to appear. 
10. G.-L. Cattani and G. Winskel, Profunctors, open maps and bisimulation, Mathematical Structures in Computer Science 15 (2005), no. 3, 553-614.

11. E. Cheng, M. Hyland, and J. Power, Pseudo-distributive laws, Electronic Notes in Theoretical Computer Science 83 (2003).

12. B. Day, On closed categories of functors, Reports of the Midwest Category Seminar IV, Lecture Notes in Mathematics, vol. 137, Springer-Verlag, 1970, pp. 1-38.

13. B. Day and R. Street, Monoidal bicategories and Hopf algebroids, Advances in Mathematics 129 (1997), 99-157.

14. _ L L L monoids, pseudo-operads, and convolution, Diagrammatic morphisms and applications (San Francisco, CA, 2000), Contemporary Mathematics, vol. 318, American Mathematical Society, 2003, pp. 75 - 96.

15. T. Ehrhard and L. Reigner, The differential lambda calculus, Theoretical Computer Science 309 (2003), no. 1-3, 1-41.

16. S. Eilenberg and G. M. Kelly, Closed categories, Proceedings of La Jolla Conference on Categorical Algebra, Springer-Verlag, 1966, pp. 421-562.

17. M. Fiore, Mathematical models of computational and combinatorial structures, Invited address for Foundations of Software Science and Computation Structures (FOSSACS 2005), volume 3441 of Lecture Notes in Computer Science, pages 25-46. SpringerVerlag, 2005.

18. M. Fiore, N. Gambino, M. Hyland, and G. Winskel, Two-dimensional Kleisli structures, in preparation.

19. N. Gambino, On the coherence conditions for pseudo-distributive laws, Draft, 2006.

20. J.-Y. Girard, Linear logic, Theoretical Computer Science 50 (1987), 1-101.

21. Normal functors, power series and $\lambda$-calculus, Annals of Pure and Applied Logic 37 (1988), 129-177.

22. G. B. Im and G. M. Kelly, A universal property of the convolution monoidal structure, Journal of Pure and Applied Algebra 43 (1986), 75-88.

23. A. Joyal, Une théorie combinatoire des séries formelles, Advances in Mathematics 42 (1981), 1-82.

24. - Foncteurs analytiques et espèces de structures, Combinatoire Énumérative, Lecture Notes in Mathematics, vol. 1234, Springer-Verlag, 1986, pp. 126-159.

25. G. M. Kelly, On the operads of J.P. May, Reprints in Theory and Applications of Categories (2005), no. 13, 1-13. Reprint of a manuscript from 1972.

26. C_ Coherence theorems for lax algebras and for distributive laws, Category Seminar (Proc. Sem., Sydney, 1972/1973), Lecture Notes in Mathematics, vol. 420, Springer, 1974, pp. $281-375$.

27. , On clubs and doctrines, Category Seminar (Proc. Sem., Sydney, 1972/1973), Lecture Notes in Mathematics, vol. 420, Springer, 1974, pp. $181-256$.

28. On clubs and data-type constructors, Applications of Categories in Computer Science, LMS Lecture Notes, vol. 177, Cambridge University Press, 1992, pp. 163-190.

29. J. L. Krivine, Lambda-calculus, types and models, Series in computers and their applications, Ellis Horwood, 1993.

30. J. Lambek and P. Scott, Introduction to higher order categorical logic, Cambridge studies in advanced mathematics, vol. 7, Cambridge University Press, 1986.

31. F. W. Lawvere, Metric spaces, generalized logic, and closed categories, Rend. del Sem. Mat. e Fis. di Milano 43 (1973), 135-166.

32. T. Leinster, Higher operads, higher categories, Cambridge University Press, 2004.

33. S. MacLane, Categories for the working mathematician, second ed., Springer-Verlag, 1998.

34. F. Marmolejo, Distributive laws for pseudo-monads, Theory and Applications of Categories 5 (1999), no. 5, $91-147$.

35. J. P. May, The geometry of iterated loop spaces, Lecture Notes in Mathematics, vol. 271, Springer, 1972. 
36. P.-A. Melliès, Categorical models of linear logic revisited, Theoretical Computer Science (to appear). Available from $\langle$ http://www.pps.jussieu.fr/ $/$ mellies $/\rangle$.

37. M. Méndez, Species on digraphs, Advances in Mathematics 123 (1996), 243-275.

38. M. Méndez and O. Nava, Colored species, c-monoids and plethysm, I, Journal of Combinatorial Theory, Series A 64 (1993), 102-129.

39. J. Morton, Categorified algebra and quantum mechanics, Available from 〈http:// arxiv.org/abs/math.QA/0601458/〉, 2006.

40. O. Nava and G.-C. Rota, Plethysm, categories, and combinatorics, Advances in Mathematics 58 (1985), 61-88.

41. A. Schalk, What is a categorical model of linear logic?, Notes for research students. Available from $\langle$ http://www.cs.man.ac.uk/ schalk/ $\rangle, 2004$.

42. D. Scott, Relating theories of the $\lambda$-calculus, To H.B. Curry: Essays on combinatory logic, lambda calculus and formalism, Academic Press, 1980, pp. 403-450.

43. R. Street, Fibrations and Yoneda's lemma in a 2-category, Category Seminar (Proc. Sem., Sydney, 1972/1973), Lecture Notes in Mathematics, vol. 420, Springer, 1974, pp. $104-133$

44. _ Fibrations in bicategories, Cahiers de Topologie et Géometrie Différentielle 21 (1980), no. 2, 111-160.

Computer Laboratory, University of Cambridge, 15 JJ Thomson Avenue, CAmbridge CB3 0FD, UK.

E-mail address: Marcelo.Fiore@cl.cam.ac.uk

Laboratoire de Combinatoire et Informatique Mathématique, DÉpartements De Mathématiques et D'Informatique, Université du Québec À Montréal, Case Postale 8888, Succ. Centre-Ville, Montréal (QC) H3C 3P8, Canada.

E-mail address: gambino@math.uqam.ca

DPMMS, University of CAmbridge, Wilberforce Road, CAmbridge CB3 0WA, UK.

E-mail address: M.Hyland@dpmms.cam.ac.uk

Computer Laboratory, University of Cambridge, 15 Jj Thomson Avenue, CAmbridge CB3 0FD, UK.

E-mail address: Glynn.Winskel@cl.cam.ac.uk 